\title{
Blocages et Stratégies en expression orale : le cas des chypriotes hellénophones FLE
}

\author{
Christoforou, Nathalie, \& Kakoyianni-Doa, Fryni \\ Université de Chypre \\ \{christoforou.c.nathalie et frynidoa\}@ucy.ac.cy
}

\section{Introduction}

L'expression orale demeure une compétence où l'on retrouve un nombre important de blocages chez les apprenants de langues étrangères. Comme l'affirment Cuq et Gruca (2005, p. 179) l'association « entre le verbal et le gestuel, les traits émotionnels et l'implicite que véhicule l'oral et toutes les formes d'interaction sont autant de facteurs qui complexifient le domaine et peuvent être sources de blocage pour un étudiant étranger ». Les deux auteurs montrent clairement que la variété des composantes de l'expression orale peut être en effet à l'origine de blocages. Pour résoudre cette difficulté, de nombreuses recherches ${ }^{1}$ proposent l'emploi de stratégies de communication. Selon Bialystok (1990, p. 1), « l'aisance et la fluidité avec lesquelles on navigue d'une idée à une autre dans sa langue maternelle, sont constamment brisées par une lacune dans sa connaissance d'une langue seconde. Les lacunes/blocages peuvent prendre plusieurs formes - d'un mot, d'une structure, d'une phrase, d'un marqueur de temps, d'un idiome. Les efforts de surmonter ces lacunes s'appellent des stratégies de communication ». Il nous semble de ce fait primordial de nous arrêter sur le rôle des stratégies de communication dans le déblocage de la parole. Ainsi, la question qui se pose est de savoir si l'enseignement-apprentissage des stratégies de communication, voire des «efforts de surmonter [des] lacunes » (ibid.) (formuler autrement, utiliser des indices physiques etc.) améliore la performance des apprenants et contribue au déblocage de la parole. Afin d'examiner cela, il est nécessaire de porter une attention particulière sur la progression au niveau des blocages survenus chez notre public, apprenants chypriotes hellénophones de Français Langue Étrangère (FLE), ainsi que sur les stratégies de communication employées pour résoudre ces blocages en expression orale. Dans un premier temps de la présente étude nous exposerons notre cadre théorique ainsi que notre méthodologie de recherche et par la suite notre expérimentation avec ses résultats.

\section{Cadre théorique et méthodologie de recherche}

\subsection{Stratégies communicatives pour le déblocage de la parole}

Il est indéniable que différents facteurs influent sur la communication et le blocage des apprenants. L'apprentissage d'une langue étrangère est un processus unique dans son genre car les apprenants sont invités à communiquer dans une langue qu'ils ne maîtrisent pas parfaitement, dans leur propre interlangue (Selinker 1972). La psycholinguiste Dolly Young accorde une grande importance à l'influence de l'anxiété sur l'apprentissage des langues étrangères. D'après l'auteure, « des recherches ont démontré que les apprenants anxieux souffrent considérablement pendant des activités. L'anxiété a un impact négatif sur leur comportement face à l'apprentissage de la langue [...] L'oral est le code le plus cité comme étant une source d'anxiété pour les apprenants de langues étrangères » (1999, p. 125). Par ailleurs, selon Tsiplakides et Keramida (2009, p. 39) «les apprenants qui présentent une certaine appréhension communicative ne se sentent pas à l'aise de communiquer dans la langue cible devant les autres, en raison de leur connaissance limitée de la langue, notamment en expression et compréhension orales ». Il est de ce fait intéressant de noter que l'oral apparait comme une source importante d'anxiété qui fait souffrir les apprenants. Par ailleurs, le Cadre Européen Commun de Références pour les langues (CECR), précise que 
l'inhibition de l'apprenant influence fortement sa performance ainsi que l'accomplissement de la tâche. Plus précisément, il déclare

\begin{abstract}
qu'une image positive de soi et l'absence d'inhibitions contribueront au succès de l'exécution d'une tâche lorsque l'apprenant a la confiance en soi qu'il faut pour la mener à son terme. Il prendra, par exemple, le contrôle de l'interaction si nécessaire (intervenir pour demander une clarification, vérifier que le sens est compris, prendre des risques le cas échéant ou, en cas de problème de compréhension, continuer à lire ou à écouter et faire des hypothèses et des déductions, etc.) ; le degré d'inhibition peut dépendre de la situation donnée ou de la tâche. (CECR 2001, p. 122).
\end{abstract}

Afin que l'apprenant surmonte l'inhibition, le CECR accorde une place importante aux stratégies communicatives. Les stratégies «sont le moyen utilisé par l'usager d'une langue pour mobiliser et équilibrer ses ressources et pour mettre en œuvre des aptitudes et des opérations afin de répondre aux exigences de la communication en situation et d'exécuter la tâche avec succès et de la façon la plus complète et la plus économique possible - en fonction de son but précis. » (p. 48). Nous constatons que, selon le CECR, l'accomplissement réussi de la tâche dépend considérablement des stratégies de communication. Par ailleurs, le CECR ajoute qu'il existe deux types de stratégies: "les stratégies d'évitement» et « les stratégies de réalisation» (p. 53). Quelques exemples de stratégies d'évitement sont le changement du sujet de la conversation et son adéquation aux moyens d'expression dont dispose l'apprenant. Les stratégies de réalisation élèvent le niveau de la tâche car l'apprenant fait de son mieux pour transmettre le message et poursuivre la communication. Quelques exemples donnés des stratégies de réalisation par le CECR sont les "approximations et généralisations sur un discours simplifié, [les] paraphrases ou descriptions de ce que l'on veut dire, et même [les] tentatives de "francisation » d'expressions de la L1 » (ibid.). Il est à noter que des classements similaires apparaissent également chez d'autres auteurs beaucoup plus tôt, à savoir dans les travaux de Faerch et Kasper (1983). Les deux auteurs parlent des stratégies de réduction et d'accomplissement. Selon eux, «l'apprenant qui tente de résoudre le problème en élargissant ses ressources communicatives» (1983, p. 52-3) utilise des stratégies d'accomplissement. En revanche, l'apprenant qui «communique à travers des moyens d'un système « réduit» afin d'éviter de produire des occurrences incorrectes»(ibid.) emploie des stratégies de réduction formelle et celui qui «réduit son but communicatif afin d'éviter le problème » (ibid.) emploie des stratégies de réduction fonctionnelle.

Une autre auteure qui attribue aux stratégies une importance majeure dans la résolution de problèmes est Oxford $(1990,2011)$. Rebecca Oxford, qui nous a le plus inspirées pour la présente étude, considère que les apprenants autonomes "utilisent les stratégies de manière interactive et dynamique pour gérer leur apprentissage » $(2011$, p. 7). Ils participent ainsi «activement dans leur propre apprentissage et atteignent des objectifs variés » (p. 15). Cette auteure présente un classement qui diffère considérablement des autres, car elle inclut ce qui est communément appelé les stratégies communicatives dans les stratégies socioculturelles-interactives qui «facilitent directement la communication et la compréhension approfondie du contexte socioculturel ainsi que des rôles adoptés à l'intérieur de ce contexte » (p. 88). De plus, elle considère que le fait de surmonter des lacunes à l'oral est une seule stratégie manifestée par le biais de diverses tactiques «des manifestations précises d'une stratégie » (p. 31), à savoir des moyens précis de communication. Les exemples de tactiques ${ }^{2}$ ou de moyens de communication présentés par Oxford pour surmonter des blocages en expression orale consistent en général à : «formuler autrement, utiliser des indices physiques, prétendre comprendre et changer le sujet de la conversation» (p. 134). Plus particulièrement «formuler autrement » est manifesté 1) en inventant un mot, 2) en décrivant l'idée qu'un mot exprime : de quoi cela a l'air, à quoi cela sert, si on peut le porter, le manger ou le boire (une circonlocution), 3) en employant des synonymes, 4) en employant des antonymes (ce n'est pas cela mais le contraire) ou 5) en s'exprimant différemment. Utiliser des indices physiques apparait en 1) faisant des gestes et 2) adoptant une expression embarrassée pour exprimer le besoin d'aide. Prétendre comprendre se présente par le hochement de tête de l'apprenant qui veut montrer qu'il a compris et éviter ainsi de parler. Enfin changer le sujet de conversation est manifesté lorsque l'apprenant en choisit un autre mieux maitrisé. Le tableau 1 en fin de document que nous avons fabriqué est fortement inspiré des tactiques proposées par Oxford (2011). Il est à noter que l'auteur ne se concentre 
pas sur la distinction entre les stratégies de réduction et celles d'accomplissement (ni sur celles d'évitement et de réalisation). Or, elle les présente comme des tactiques faisant partie d'une seule stratégie socioculturelle-interactive. Il est ainsi clair que le classement effectué par Oxford n'insiste pas sur la distinction entre les stratégies de réduction et d'accomplissement car, pour elle, toutes les tactiques mentionnées plus haut contribuent au déblocage de la parole. En outre, Oxford propose des solutions aux blocages également par l'intermédiaire des stratégies affectives : 1) «l'activation d'émotions, croyances et attitudes de soutien » et 2) «l'engendrement et le maintien de la motivation» (p. 64). Nous donnons des exemples de tactiques socioculturelles-interactives et affectives au tableau 1 en fin de document.

Notre méthodologie de recherche se fonde ainsi fortement sur le classement proposé par Oxford (2011), qui accorde une importance considérable au déblocage de la parole, par le biais de tactiques socioculturelles-interactives et affectives. Néanmoins, nous considérons que l'auteure n'approfondit pas suffisamment sur les moyens de communication non verbaux. Comme précité, Oxford propose deux seuls exemples de tactiques qui consistent à utiliser des indices physiques : 1) faire des gestes et 2) adopter une expression embarrassée pour exprimer le besoin d'aide. Par conséquent, afin d'avoir recours à un nombre plus important de tactiques d'utilisation d'indices physiques, nous nous fondons également sur la théorie de McNeill (1992) qui étudie le non verbal en profondeur. McNeill (1992) distingue la pantomime des gestes et définit différents types de gestes. Il insiste fortement sur la distinction entre les gestes «qui ne surviennent jamais sans paroles » et la pantomime où « la parole n'est pas obligatoire » (1992, p. 37). Dans cette optique, nous distinguons deux différentes tactiques pour résoudre un blocage communicatif en expression orale, 1) la pantomime et 2) les gestes. De plus, il est tout aussi important de prendre en compte qu'il existe divers types de gestes. McNeill définit entre autres le geste iconique ou illustratif comme ayant « un lien direct au contenu semantique de la parole» (p. 12), le geste déictique qui consiste à pointer et le geste métaphorique qui apparait lorsqu'il d'agit d'illustrer "plutôt une idée plus abstraite qu'un objet ou événement concret» (p. 14). Les différents types de gestes présentés par McNeill ${ }^{3}$ nous ont servi de référence pour le classement des gestes employés par nos apprenants dans le but de se débloquer. Nous donnons des exemples de gestes effectués par des apprenants dans la partie 3.3.

Pour cette étude, nous adoptons le classement d'Oxford (2011) qui expose un nombre important de tactiques de déblocage de la parole en expression orale et celui de McNeill (1992) qui définit différents types de gestes. Nous nous inspirons également de la distinction précitée entre les stratégies de réduction et celles d'accomplissement de Faerch et Kasper (1983). Nous avons effectué aussi ce choix car nous considérons qu'il est important de déterminer si nos apprenants ont tendance à abandonner, partiellement ou totalement, la tâche (stratégies de réduction) ou à poursuivre leurs efforts de formulation et reformulation (stratégies d'approfondissement).

Ainsi, dans la présente étude, afin de réaliser si l'enseignement-apprentissage des tactiques améliore la performance des apprenants et résout le blocage de la parole nous avons mené une étude en deux phases. Nous avons premièrement cherché à savoir ce que les apprenants chypriotes hellénophones pensent de leur performance orale en classe de FLE et plus particulièrement de la place et des raisons de leurs blocages en expression orale. Nous avons ainsi réalisé une première étude (des observations de classe et entretiens) fondée sur les représentations des apprenants. Par la suite nous avons créé un atelier d'expression orale centré sur le déblocage de la parole, par le biais de l'enseignement-apprentissage d'un nombre choisi de tactiques. Enfin, nous présentons les résultats d'une expérimentation au sein de l'atelier visant à mettre en évidence le taux des blocages des apprenants lors d'une activité d'expression orale et notamment la progression des apprenants à la fin de l'atelier.

\section{2 Étude sur les représentations des apprenants}

Les observations de classe nous ont permis de réaliser que les apprenants donnent l'impression d'être inhibés en classe de FLE et n'osent pas toujours prendre la parole. Ainsi, à travers les entretiens nous avons cherché à savoir si cela était également ressenti par les apprenants eux-mêmes et enfin pour quelles raisons cela se produisait. Plus particulièrement, la première phase de notre étude a été réalisée dans le but de mieux contextualiser notre recherche et avoir ainsi une représentation générale de la présence des 
blocages chez les apprenants chypriotes hellénophones. Nous sommes entrées dans des classes de FLE et nous avons observé 30 heures d'enseignement soit 14 groupes différents composés au total de 230 apprenants, dans des écoles publiques, un institut privé et à l'université de Chypre. De plus, nous avons tenté de mettre en évidence un taux plus ou moins représentatif des blocages, en réalisant des entretiens avec des apprenants de FLE. En janvier 2013, nous avons pu interviewer 60 apprenants, de 12 à 22 ans, de niveaux A1-B1 du CECR. Nous nous sommes fondées sur un questionnaire de 20 questions ${ }^{4}$, dans la visée de mieux nous rendre compte des croyances et représentations des apprenants concernant leur performance en classe, en expression orale.

Pour la présente étude, parmi les 20 questions, nous n'en avons retenu que 3 concernant précisément le blocage des apprenants. La première question posée aux apprenants interviewés était la suivante : «Participez-vous en classe en français? ». La graphique 1 en fin de document représente clairement le pourcentage de chaque réponse : 40 apprenants $(67 \%)$ ont répondu négativement, 11 apprenants (18\%) ont répondu positivement et 9 apprenants (15\%) affirment participer quelquefois en classe en français. La deuxième question est : «Êtes-vous bloqués lorsque vous parlez en français? ». Les réponses des apprenants sont de nouveau révélatrices (graphique 2 en fin de document). 52 apprenants $(87 \%)$ ont répondu positivement et $8(13 \%)$ ont répondu «quelquefois ». Il est en effet très intéressant de noter qu'aucun apprenant n'a répondu «non » à cette question. Enfin, la troisième question porte sur les raisons des blocages : «Quelles sont à votre avis les principales raisons de votre blocage ?» (graphique 3 en fin de document). Nous avons reçu 6 réponses, à savoir 6 raisons différentes de blocage en expression orale, rencontrées au total 84 fois $^{5}$. Nous avons relevé 12 fois (14\%) la peur de commettre des erreurs, 15 fois (18\%) l'anxiété, 8 fois $(10 \%)$ la honte face à ses camarades, 20 fois $(24 \%)$ la difficulté de trouver le bon mot, 22 fois $(26 \%)$ de bien prononcer et 7 fois $(8 \%)$ de ne pas commettre des erreurs grammaticales.

Par conséquent, les résultats de cette étude mènent à conclure : 1) que les apprenants, dans leur grande majorité, ne participent pas en classe en français, 2) qu'ils considèrent être bloqués en expression orale et 3) que les raisons les plus importantes de blocage pour les chypriotes hellénophones en expression orale de FLE sont l'anxiété et les difficultés en ce qui touche la prononciation et le vocabulaire. Ces résultats mettent en évidence la nécessité d'un enseignement spécialisé sur les stratégies de communication, voire l'utilisation de tactiques de déblocage de la parole. Nous avons ainsi procédé à l'élaboration d'un atelier d'expression orale.

\section{Atelier d'expression orale}

\subsection{Public et objectifs de l'atelier}

L'atelier d'expression orale d'une durée de 18 heures, s'étale de février à mai 2013. L'atelier compte 21 participants, étudiants du Département d'Études françaises et de Langues vivantes de l'université de Chypre, de première et deuxième années des programmes en études françaises et européennes, de niveaux A2-B2 du CECR. Les 21 participants sont séparés dans trois groupes de 6, 7 et 8 participants. Les séances sont enregistrées et les activités d'expression orale sont également filmées puis transcrites. L'atelier est facultatif, sans évaluation et est fondé sur trois objectifs de base. Premièrement, l'apprentissage du français langue-culture à travers l'utilisation consciente des stratégies socioculturelles-interactives, comme elles étaient citées auparavant. Deuxièmement, l'entrainement à l'utilisation de ces stratégies afin de parvenir à un emploi conscient et autonome par les apprenants. Troisièmement, la sensibilisation à l'emploi des stratégies affectives comme elles sont présentées par Oxford (2011). Certains principes de base de l'atelier sont le développement d'une ambiance ludique, sécurisante et amicale, en accordant de l'importance à la place de l'humour et à la déculpabilisation de l'erreur. Nous prenons également en compte les facteurs affectifs influençant la performance des apprenants, comme les expériences personnelles, les croyances et attitudes. 


\subsection{Questionnaire/Portfolio}

A la première séance de l'atelier nous avons demandé aux apprenants de compléter un questionnaire (tableau 1 en fin de document), que nous avons fabriqué, étant inspirées des stratégies et tactiques proposées par Oxford (2011). Le questionnaire avait un double objectif: sensibiliser les apprenants aux différentes tactiques de communication et étudier la fréquence des tactiques par chaque apprenant, afin de mieux connaitre les participants de l'atelier. Nous avons séparé le questionnaire en trois parties. La première partie (partie A du tableau 1 en fin de document) se concentre sur les tactiques socioculturellesinteractives qui contribuent au déblocage de la parole. Nous proposons des tactiques comme l'utilisation de gestes, de synonymes, de mots différents, de phrases plus simples etc. Dans la deuxième partie du questionnaire (partie B du tableau 1 en fin de document) nous présentons des tactiques socioculturellesinteractives qui concernent plus la coopération lors d'une interaction que le déblocage de la parole, par exemple demander une clarification ou pratiquer la langue avec des natifs. Dans la troisième partie (partie $\mathrm{C}$ du tableau 1 en fin de document) nous avons rassemblé des tactiques affectives contribuant au déblocage de la parole, comme utiliser la relaxation et la méditation ou prendre des risques même s'il est possible de commettre des erreurs.

Le questionnaire a été aussi utilisé tout au long de l'atelier en tant que portfolio. Selon le CECR, «le portfolio permet à un apprenant d'enregistrer et de présenter différentes facettes de sa biographie langagière » (2001, p. 132). Nous avons ainsi insisté sur son utilisation pendant des moments de blocage, afin d'étudier les résultats d'une approche consciente d'apprentissage des stratégies et tactiques communicatives. Ce portfolio centré sur l'utilisation des stratégies de déblocage de la parole a joué un rôle majeur dans la progression des apprenants. A plusieurs reprises, nous avons pu filmer des moments où des apprenants bloqués, surmontent le blocage après la consultation du portfolio. Dans les enregistrements filmés, nous pouvons voir les apprenants chercher un mot, prendre quelques secondes pour réfléchir, ensuite consulter le portfolio et utiliser une tactique qui résout le problème. De cette manière, les apprenants se débloquent plus rapidement et trouvent un remède afin de poursuivre la communication.

\subsection{Approche de l'atelier et premiers résultats}

Dans l'atelier d'expression orale, nous suivons un plan de travail composé de quatre phases. Nous commençons toujours les séances par des activités de relaxation et de respiration afin de libérer les apprenants de toute anxiété, les sensibiliser à l'utilisation des stratégies affectives et offrir à l'atelier une ambiance plus ludique. Pendant les trois premières séances, nous avons également eu recours à des activités ou des jeux de collaboration centrés sur le développement de la confiance au sein du groupe. A la suite de ces activités, nous consacrons toujours vingt minutes à des activités de phonétique corrective, de dynamisation de la voix ainsi qu'à l'utilisation active des gestes et expressions du visage. Dans un troisième temps, nous procédons à des activités variées ayant comme but le développement de l'utilisation consciente et autonome des tactiques pour surmonter des blocages. Les activités d'expression orale prennent la forme de différentes techniques : des dramatisations, improvisations, débats, jeux de rôles etc. Par ailleurs, les activités filmées, servent de support d'autoévaluation aux apprenants. Nous avons téléchargé des parties sélectionnées des activités filmées sur la plateforme numérique de l'université, de manière à ce que chaque participant puisse commenter et évaluer sa propre performance ${ }^{6}$. Les apprenants, conscients de l'objectif de l'atelier, proposent souvent dans leurs commentaires des tactiques différentes, par exemple : «Je pouvais faire un geste pour le mot avion» (apprenant A), ou « Pour le mot discrimination je pouvais dire racisme, n'est-ce pas ? » (apprenant B).

Afin d'étudier le progrès langagier des étudiants, nous avons mis en place une expérimentation, permettant de nous rendre compte du taux réel de blocages chez les participants, au début et à la fin de l'atelier. Un nombre de 13 étudiants (les deux des trois groupes de l'atelier) ont participé aux activités d'expression orale de l'expérimentation, qui consistaient à décrire deux images de leur choix, dire pourquoi elles étaient sélectionnées et enfin parler des sentiments éprouvés en les regardant. Il est à noter que des images différentes ont été choisies au début et à la fin de l'atelier. Les deux activités étaient 
filmées et transcrites et représentent des corpus de 1029 mots au début du semestre et 1231 mots à la fin. Les résultats sont particulièrement intéressants. La première fois, au début de l'atelier, nous avons pu relever 34 blocages exprimés par de longues pauses, de plus de 6 secondes, accompagnées souvent d'expressions embarrassées et d'hésitations « euh ». Nous n'avons pas pris en compte les pauses moins longues de 6 secondes qui nous ont plutôt parues comme des hésitations plus ou moins naturelles. La deuxième fois, à la fin de l'atelier, nous n'avons relevé que 12 blocages.

Il est intéressant d'aller encore plus en profondeur dans l'analyse des blocages (cf. tableau 2 en fin de document) et constater que la première fois 12 blocages sont restés sans résolution et n'étaient pas suivis de tactique socioculturelle-interactive de déblocage de la parole, avec comme résultat l'abandon du message, à savoir l'emploi de stratégies de réduction fonctionnelle qui consistent pour Faerch et Kasper (1983, p. 52-3) à réduire le «but communicatif afin d'éviter le problème ». En revanche, à la fin du semestre, parmi les 12 blocages relevés, aucun n'est resté sans résolution, sans recours à une stratégie. Comme nous pouvons le voir au tableau 2, la première fois, un participant a eu recours à sa langue maternelle, le grec, et la deuxième fois, 3 participants ont eu recours 2 fois au grec et 1 fois à l'anglais. Ces 4 productions étaient présentées sous forme de question, que nous avons classées également en tant que demande de confirmation. Par exemple : «Ici je suis à un euh show?». Par ailleurs, nous avons relevé un geste déictique (le participant pointait vers en bas) la première fois accompagnant une tactique d'emploi d'un mot différent « le café euh tombe » au lieu de «le café est renversé ». A la fin de l'atelier, nous avons noté un geste illustratif pour le mot « entourée » dans l'acte de parole donné par l'apprenant «ma vie est entourée de la mer et des animaux ». Au début, nous avons également noté 5 fois où les participants ont trouvé un synonyme ou le mot qu'ils cherchaient au départ (nous ne pouvons pas être sûres s'il s'agit du mot recherché ou d'un synonyme) et 4 fois à la fin. La première fois nous avons compté 10 tactiques d'emploi de circonlocutions, à savoir des descriptions d'une idée ou un objet et une seule fois à la fin. Des circonlocutions intéressantes utilisées étaient les suivantes : "c'est euh dans ce qu'on boit le café » pour le mot tasse, « euh le téléphone l'a derrière pour connecter sur euh il est spiral et il est derrière » pour le mot fil et « des choses qu'on met le feu » pour le mot allumettes. Il est à noter que 3 fois les circonlocutions étaient accompagnées de la tactique d'emploi de mots passe-partout comme "quelquechose ", " une chose », " un truc » comme dans l'exemple de la circonlocution suivante: «derrière il y a quelquechose qui forme une pâte » utilisée pour expliquer le mot queue. De plus, nous avons noté 3 tactiques d'utilisation de mots différents la première fois et 2 la deuxième fois comme « stylos » au lieu d'allumettes, « un carré » au lieu d'un rectangle. Enfin, nous avons relevé 2 tactiques de simplification de la phrase au début et encore 2 à la fin comme « euh il donne un euh bisous » au lieu de «il embrasse ».

Nous constatons ainsi que pendant le semestre les participants de l'atelier ont pu véritablement travailler sur le déblocage de la parole et la résolution de ces blocages à travers des tactiques diverses. Les résultats les plus remarquables sont la diminution claire de blocages de 34 à 12, ainsi que la disparition de blocages non résolus avec abandon du message (de 12 à 0 ). La diminution de l'utilisation de la tactique de circonlocution de 10 à 1 après un blocage, peut à notre avis s'expliquer par le fait que les apprenants à la fin du semestre parlaient plus du symbolisme des images et des sentiments qu'ils éprouvaient en les regardant sans rester longtemps sur leur description.

Les résultats de l'atelier sont très positifs. Nous avons demandé aux apprenants de s'exprimer anonymement sur cette nouvelle expérience. Nous retenons quatre réponses révélatrices des résultats de l'atelier et soulignons les éléments les plus intéressants :

C'était une très bonne expérience. J'ai amélioré mon français et surtout ma prononciation. J'ai appris des expressions de la vie quotidienne et découvert des façons de m'exprimer que je ne connaissais pas. Nous devons continuer l'atelier. (Apprenant A).

Nous avons amélioré notre vocabulaire. (Apprenant B). 
Nous avons appris à mieux communiquer en français et à être plus sociables. C'était une expérience formidable! (Apprenant C).

C'était une très bonne expérience. J'ai pu améliorer mon accent et j'ai surmonté ma honte et ma gêne quand je suis devant un public. En plus, nous avons trouvé des moyens de remplacer un mot quand nous ne le trouvons pas. Ce serait bien d'avoir l'atelier le semestre prochain. (Apprenant D).

Les étudiants reconnaissent tous l'importance de l'atelier. A la fin de l'expérience, ils se sentent plus à l'aise, en ayant plus de confiance en eux. Ils ont découvert des moyens de communiquer, à savoir des tactiques, leur permettant de trouver des solutions à des blocages. Ils considèrent avoir amélioré leur prononciation, vocabulaire et expression orale.

\section{Conclusion}

Les résultats de l'étude mettent en évidence d'une part la place importante des blocages en expression orale du Français Langue Étrangère et d'autre part l'apport de l'enseignement-apprentissage des stratégies de communication contribuant au déblocage de la parole. Nous retenons six résultats :

1) Les apprenants considèrent être bloqués en expression orale notamment à cause : a) de la prononciation, b) du vocabulaire et c) de l'anxiété.

2) L'enseignement-apprentissage des stratégies de communication aide les apprenants à réaliser qu'il existe des stratégies facilitant la communication.

3) L'enseignement-apprentissage conscient des stratégies de communication participe au développement de la confiance en soi de l'apprenant, de manière à ce qu'il soit capable de mieux gérer des situations de blocage.

4) L'enseignement-apprentissage des stratégies de communication peut conduire à une utilisation plus performante de celles-ci par les apprenants, en améliorant ainsi la compétence d'expression orale.

5) Le nombre de blocages peut baisser considérablement à la fin d'un enseignement ciblé sur le déblocage de la parole et l'apprentissage des stratégies et tactiques socioculturellesinteractives.

6) L'abandon du message après un blocage peut diminuer considérablement voire même disparaître à la fin d'un tel enseignement-apprentissage.

L'étude conduit à conclure qu'il est nécessaire de reconsidérer la place accordée, en classe de langue étrangère, à l'enseignement-apprentissage des stratégies de communication, facilitant le processus d'acquisition. Il est d'une grande importance que les apprenants réalisent que les stratégies étudiées constituent un vrai remède aux blocages et contribuent au développement de leur autonomie. C'est pourquoi, l'expression orale requiert le développement d'un apprentissage conscient des stratégies communicatives, impliquant le besoin d'un enseignement systématique. 


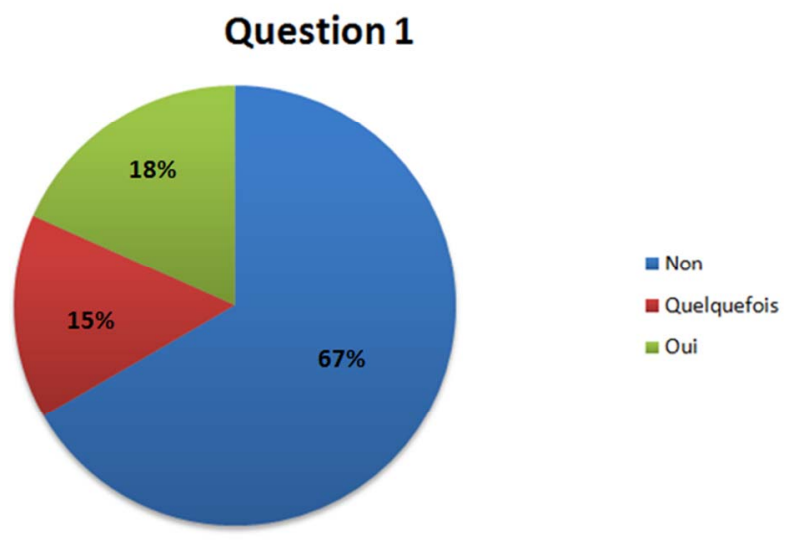

Graphique 1 : Réponses des apprenants à la question : «Participez-vous en classe en français ? »

\section{Question 2}

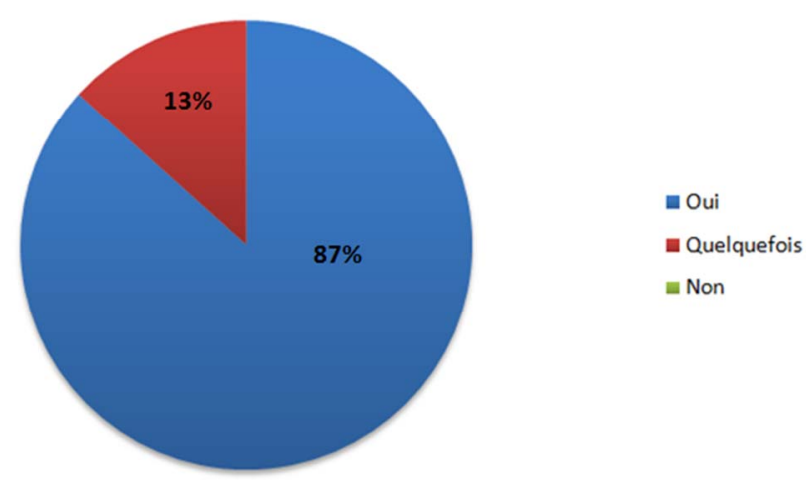

Graphique 2 : Réponses des apprenants à la question : «Etes-vous bloqués lorsque vous parlez en

\section{Question 3}

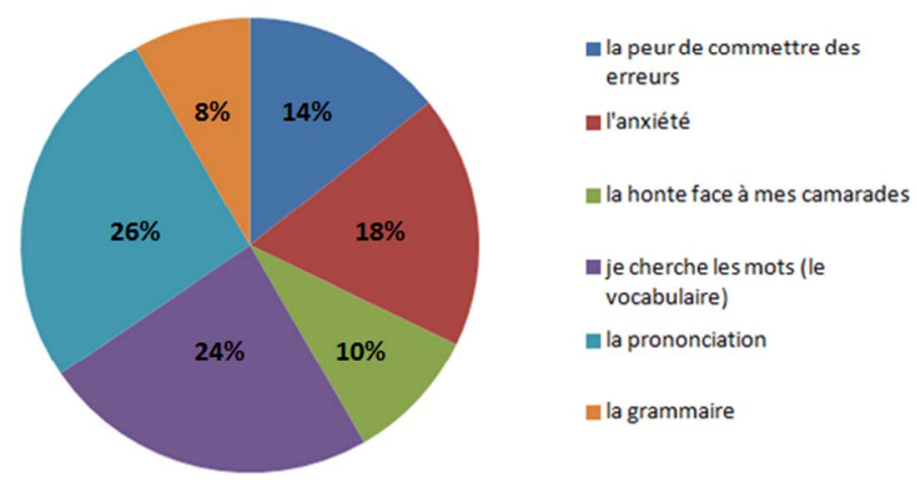

Graphique 3 : Réponses des apprenants à la question : «Quelles sont à votre avis les principales raisons de votre blocage? » 


\section{QUESTIONNAIRE}

Quelles sont vos stratégies d'expression orale? Cochez la case qui correspond à la fréquence d'utilisation de chaque stratégie.

$1=$ jamais, 2 = rarement, $3=$ quelquefois, $4=$ souvent et $5=$ toujours

\begin{tabular}{|c|c|c|c|c|c|c|}
\hline & $\begin{array}{l}\text { Partie A : Tactiques socioculturelles-interactives. } \\
\text { Pour surmonter des lacunes/blocages à l'oral }\end{array}$ & 1 & 2 & 3 & 4 & 5 \\
\hline 1 & Je passe à ma langue maternelle ou à une langue partagée avec l'interlocuteur. & & & & & \\
\hline 2 & Je demande qu'on m'aide : « comment dit-on... ? » « comment appelle-t-on... ?». & & & & & \\
\hline 3 & $\begin{array}{l}\text { Je fais savoir que j'ai besoin d'aide, par exemple en marquant une pause ou en adoptant une } \\
\text { expression embarrassée. }\end{array}$ & & & & & \\
\hline 4 & Je mime, je fais des gestes ou j'utilise les jeux d'expression faciale. & & & & & \\
\hline 5 & J'évite la communication partiellement ou totalement. & & & & & \\
\hline 6 & Je choisis le sujet de conversation. & & & & & \\
\hline 7 & $\begin{array}{l}\text { J'essaie d'amener le locuteur natif à conduire la conversation en posant des questions telles que } \\
\text { «Qu'en pensez-vous?». }\end{array}$ & & & & & \\
\hline 8 & J'évite les sujets ou idées qui pourraient me poser de trop grosses difficultés. & & & & & \\
\hline 9 & J'essaie d'utiliser une formulation plus simple, par exemple avec des phrases simples et courtes. & & & & & \\
\hline 10 & J'utilise des mots différents comme crayon au lieu de stylo. & & & & & \\
\hline 11 & $\begin{array}{l}\text { J'invente un mot en prononçant l'équivalent dans ma langue maternelle ou dans une autre } \\
\text { langue que je connais, mais avec un accent français. }\end{array}$ & & & & & \\
\hline 12 & $\begin{array}{l}\text { J'utilise des synonymes, par exemple un mot qui a un sens presque identique: bateau au lieu de } \\
\text { navire. }\end{array}$ & & & & & \\
\hline 13 & J'emploie des mots passe-partout comme truc ou machin. & & & & & \\
\hline 14 & $\begin{array}{l}\text { Je décris l'idée qu'un mot exprime : de quoi cela a l'air, à quoi cela sert, si on peut le porter, le } \\
\text { manger ou le boire, etc. }\end{array}$ & & & & & \\
\hline 15 & $\begin{array}{l}\text { Lorsque je me rends compte d'avoir commis une erreur, je m'auto-corrige et continue } \\
\text { naturellement. }\end{array}$ & & & & & \\
\hline 16 & $\begin{array}{l}\text { J'utilise des expressions telles que : « Enfin, attendez, laissez-moi réfléchir, en fait, pas du tout, } \\
\text { absolument », pour gagner du temps. }\end{array}$ & & & & & \\
\hline & Autres: & & & & & \\
\hline
\end{tabular}




\section{Partie B : Tactiques socioculturelles-interactives}

\section{-Pour poser des questions}

17 Je demande une clarification pour comprendre ou vérifier ma compréhension de l'interlocuteur, par exemple : je demande de paraphraser, de répéter, de parler plus lentement, de donner des exemples ou d'expliquer.

Je demande à l'interlocuteur de me corriger.

-Pour coopérer avec les autres en expression orale.

19 Je m'entraine avec d'autres apprenants.

20 Je m'entraine avec des locuteurs natifs.

\section{-Pour cultiver l'empathie}

21 J'essaie de m'ouvrir à la culture de l'autre afin de mieux le comprendre.

22 J'essaie de me sensibiliser aux sentiments et aux pensées de l'autre, par exemple en observant ses comportements.

\section{Partie C: Tactiques affectives}

-Pour diminuer mon anxiété

23 J'utilise la relaxation, la respiration profonde ou la méditation.

24 J'utilise la musique, par exemple je pense à une chanson que j'aime.

25 J'utilise l'humour et le rire, par exemple l'auto-sarcasme.

\section{-Pour m'auto-encourager}

26 Je me félicite après une bonne performance en expression orale.

27 Je prends des risques même s'il est possible de commettre des erreurs.

28 Je me récompense après une bonne performance : je m'offre un cadeau.

\section{-Pour prendre mon pouls émotif}

29 J'écoute mon corps: je fais attention aux signes donnés comme le stress, la tension, l'inquiétude, la peur, la colère...

30 J'utilise une liste de contrôle : afin de découvrir les sentiments, attitudes et motivations pour l'apprentissage.

31 Je tiens un journal intime où je décris ce que je ressens lors de l'apprentissage et l'expression orale.

32 Je partage mes sentiments en expression orale avec une autre personne (enseignant, camarade, etc.)

Tableau 1 : Questionnaire et portfolio de l'atelier d'expression orale. 


\begin{tabular}{|l|l|l|}
\cline { 2 - 3 } \multicolumn{1}{l|}{} & $\begin{array}{l}\text { Activité au début du } \\
\text { semestre }\end{array}$ & Activité à la fin du semestre \\
\hline Participants & 13 & 13 \\
\hline Corpus & 1029 mots & 1231 mots \\
\hline Blocages & 34 & 12 \\
\hline Blocages avec abandon du message & 12 & 0 \\
\hline Recours au grec ou à l'anglais & 1 & 3 \\
\hline Utilisation de gestes & 1 geste déictique & 1 geste illustratif \\
\hline Demande de confirmation & 3 & 5 \\
\hline Demande d'aide & 0 & 1 \\
\hline $\begin{array}{l}\text { A finalement trouvé le mot ou a donné } \\
\text { un synonyme } \\
\text { (nous ne pouvons pas être sûres si le } \\
\text { mot prononcé est un synonyme ou le } \\
\text { mot recherché) }\end{array}$ & 5 & 4 \\
\hline Circonlocution (description) & 10 & 1 \\
\hline Utilisation de mots différents & 3 & 2 \\
\hline Simplification des phrases & 2 & 2 \\
\hline $\begin{array}{l}\text { Emploi de mots passe-partout comme } \\
\text { «quelquechose », « une chose », etc. }\end{array}$ & 2 & 1 \\
\hline
\end{tabular}

Tableau 2 : Résultats quantitatifs sur les blocages et les tactiques employées. 


\section{Références bibliographiques}

Arnold, J. (1999). Affect in Language Learning. Cambridge, UK: Cambridge University Press.

Bialystok, E. (1990). Communication Stratégies : A Psychological Analysis of Second-Language Use, London : Basil Blackwell.

Bogaards, P. (2007). Aptitude et affectivité dans l'Apprentissage des Langues Etrangères. Paris : Hatier / Didier.

Conseil de l'Europe. (2001). Cadre européen commun de référence pour les langues: Apprendre, enseigner, évaluer. Strasbourg.

Corder, S. P. (1983). Strategies of communication, In Faerch, C., Kasper, G. (dir.), Strategies in Interlanguage Communication, New York : Longman.

Cuq, J-P., Gruca, I. (2005). Cours de didactique du français langue étrangère et seconde, Grenoble : PUG.

Faerch, C., Kasper, G. (1983). Strategies in Interlanguage Communication, New York: Longman.

McNeill, D. (1992). Hand and Mind. What gestures reveal about thought, Chicago: The University of Chicago Press.

Oxford, R. L. (1990). Language learning strategies: what every teacher should know. Boston : Heinle \& Heinle Publishers.

Oxford, R. L. (2011). Teaching and Researching Language Learning Strategies, Harlow: Pearson Education Limited.

Selinker, L. (1972). Interlanguage, International Review of Applied Linguistics, X3, pp. 209-231.

Tarone, E. (1977). Conscious communication strategies in interlanguage: a process report, In Brown, D., Yorio, C., Crymes, R. (eds.), TESOL '77 Washington, DC: TESOL, pp.194-203.

Tsiplakides, I., Keramida, A. (2009). Helping Students Overcome Foreign Language Speaking Anxiety in the English Classroom : Theoretical Issues and Practical Recommendations, International Education Studies, 2(4), pp. 39-44.

Young, D. J. (ed.) (1999). Affect in Foreign Language and Second Language Learning: A practical guide to creating a low-anxiety classroom atmosphere, USA: McCraw-Hill Companies.

\footnotetext{
${ }^{1}$ Cf. Tarone (1977), Faerch et Kasper (1983) et Bialystok (1990).

${ }^{2}$ Oxford (2011) souligne qu'elle ne propose que des exemples et non pas la totalité des tactiques que l'on peut relever.

${ }^{3}$ Nous ne présentons pas dans la présente étude tous les types de gestes définis par McNeill (1992).

${ }^{4}$ Elaboré dans le cadre d'une thèse de doctorat.

${ }^{5}$ Souvent les apprenants donnaient plusieurs réponses.

${ }^{6}$ Chaque apprenant peut regarder uniquement sa performance, et non celle des autres.
} 